\title{
Declínio cognitivo e demência associados à doença de Parkinson: características clínicas e tratamento
}

\section{Cognitive impairment and dementia in Parkinson's disease: clinical characteristics and treatment}

\author{
Luciano Magalhães Melo ${ }^{1}$, Egberto Reis Barbosa², Paulo Caramelli ${ }^{3}$ \\ ${ }^{1}$ Médico neurologista colaborador do Grupo de Estudos dos Distúrbios do Movimento da Clínica Neurológica do Hospital das Clínicas da Faculdade de Medicina da Universidade de São Paulo (HC-FMUSP). \\ ${ }_{2}^{2}$ Professor livre-docente do Departamento de Neurologia da FMUSP e chefe do Grupo de Estudos dos Distúrbios do Movimento da Clínica Neurológica do HC-FMUSP. \\ ${ }_{3}^{3}$ Professor adjunto de Neurologia da Faculdade de Medicina da Universidade Federal de Minas Gerais e professor do Curso de Pós-Graduação em Neurologia da FMUSP.
}

Recebido: 13/10/2006 - Aceito: 20/12/2006

\begin{abstract}
Resumo
Contexto: A doença de Parkinson (DP) é uma moléstia progressiva de causa desconhecida, com características motoras que incluem tremor de repouso, rigidez, bradicinesia e alterações posturais. Mas o comprometimento cognitivo é comum mesmo nos indivíduos não dementes. Somando-se a isso, a DP associa-se a um aumento de risco de desenvolver demência. Objetivos: Esta revisão analisa a perda cognitiva associada à DP, sobre seus aspectos, fatores de risco, fisiopatologia e tratamento. Métodos: Esta revisão incorporou artigos obtidos pelo Medline. Resultados: Pacientes com DP, com ou sem demência, têm desempenho reduzido em vários testes cognitivos. Essa redução cognitiva pode ser atribuída à disfunção dos circuitos conectores da região frontal aos núcleos da base. Distúrbio cognitivo leve pode progredir para demência em certos pacientes. Identificaram-se alguns fatores de risco para o desenvolvimento de demência e revelaram-se aspectos sobre a fisiopatologia. Conclusão: As características neuropsicológicas da DP, as quais refletem predominantemente disfunção frontal, podem ser atribuídas a anormalidades do circuito frontoestriatal. Mas a fisiopatologia responsável pela demência associada à DP não está completamente compreendida. Anticolinesterásicos e memantina têm um modesto efeito contra sintomas cognitivos. Detecção precoce dos fatores de risco para o desenvolvimento de demência pode ajudar a criar estratégias preventivas.
\end{abstract}

Melo, L.M et al. / Rev. Psiq. Clín 34(4); 176-183, 2007

Palavras-chave: Demência, cognição, doença de Parkinson.

\begin{abstract}
Background: Parkinson disease (PD) is a progressive illness of undetermined cause with characteristic motor findings that include rest tremor, rigidity, bradykinesia, and postural disturbance. But cognitive impairment is common even in nondemented. In addition PD has been associated with an increased risk of developing dementia. Objectives: This review provides an analysis of the cognitive impairment associated with $\mathrm{PD}$, about its aspects, risk factors, pathophysiology, and treatment. Methods: The current review incorporates articles obtained through Medline. Results: Non-demented and demented patients with Parkinson's disease are impaired in several cognitive tasks. These impairments may be attributed to dysfunction the circuits connecting the frontal cortical regions and the basal ganglia. Mild cognitive dysfunctions may progress to dementia in some patients. Some risk factors for the development of dementia were identified and aspects of pathophysiology were disclosed. Conclusion: Neuropsychological profile of PD, which predominantly reflects frontal dysfunction, may be attributed to disruption of the frontoestriatal circuitry. But the pathophysiology underlying dementia in PD is not completely understood. Cholinesterase inhibitors and memantine have a modest effect against cognitive symptoms. Early detection of risk factors for development of dementia may help to create preventive strategies.
\end{abstract}

Melo, L.M et al. / Rev. Psiq. Clín 34(4); 176-183, 2007

Key-words: Dementia, cognition, Parkinson's disease. 


\section{Introdução}

A doença de Parkinson (DP) é tradicionalmente conhecida como moléstia com manifestações motoras e é a segunda doença neurodegenerativa mais comum em idosos, com prevalência estimada de 3,3\% no Brasil, de acordo com o estudo de Barbosa et al. (2006). Sintomas e sinais motores dela são bem conhecidos na prática clínica e incluem: tremor de repouso, bradicinesia (lentidão de movimentos), rigidez e alterações do equilíbrio. Essas manifestações são responsáveis por incapacidades física e psíquica, mas existem outras manifestações dessa doença que não devem ser ignoradas, pois também acarretam prejuízo significativo à qualidade de vida dos indivíduos acometidos, como, por exemplo: psicose, transtornos cognitivos e depressão (Tabela 1) (Thanvi et al., 2003). O objetivo deste artigo é revisar aspectos clínicos, fisiopatológicos e terapêuticos das alterações cognitivas associadas à DP.

Tabela 1. Sintomas e sinais não motores associados à doença de Parkinson

\begin{tabular}{l}
\hline Principais sintomas não motores da doença de Parkinson \\
\hline - Depressão \\
- Demência \\
- Ansiedade \\
- Alucinações, ilusões, psicose \\
- Perda de peso \\
- Transtornos do sono \\
- Disfunção autonômica \\
- Disfunção sexual \\
- Apatia
\end{tabular}

A presença de alterações cognitivas na DP foi ignorada por muito tempo. Isso ocorreu possivelmente por causa da descrição original dessa afecção feita por Parkinson (1817), na qual havia a afirmação de que o intelecto persistia inalterado e também pela menor sobrevida dos doentes, antes da introdução da levodopa (precursora da dopamina) no tratamento da moléstia (Emre e Aarsland, 2004). Entretanto, a DP associa-se a várias manifestações neuropsiquiátricas que têm considerável impacto na vida dos doentes (Bronnick et al., 2005; Weintraub et al., 2004). Déficits cognitivos eventualmente ocorrem já nas fases iniciais da DP, e nessas circunstâncias podem não ser clinicamente aparentes, mas detectáveis apenas por testes específicos.

$O$ termo demência associada à DP refere-se à demência que se desenvolve pelo menos 12 meses após a instalação das alterações motoras. Quando a demência desenvolve-se nos primeiros 12 meses de evolução da doença, preenche-se o critério para o diagnóstico de demência de corpos de Lewy (McKeith et al., 2005).

Entre as alterações cognitivas, a demência associada à DP é a manifestação mais grave e que aumenta o risco de morte (De Lau et al., 2005). A demência associada à
DP, assim como outras manifestações neuropsiquiátricas relacionadas a essa moléstia, também acarreta redução da qualidade de vida dos pacientes e até mesmo de seus cuidadores (Schrag et al., 2000; Aarsland et al., 1999). Além disso, a demência associada à DP é muito comum, com prevalência estimada por alguns estudos variando entre 20\% e 40\% (Cummings, 1988) e prevalência acumulada podendo chegar a $80 \%$, de acordo com os resultados do estudo de Aarsland et al. (2003a). Vale ressalvar que a média da idade da população examinada neste estudo era superior a 70 anos, o que pode ter contribuído para prevalência tão alta. Barbosa et al. (1987) demonstraram prevalência de demência associada à DP de 18,7\% em estudo realizado no Brasil.

A caracterização de fatores de risco para o desenvolvimento de demência e o conhecimento de alterações cognitivas precoces e das características da demência associada à DP constituem áreas de grande interesse atual, pois poderão ser úteis para a introdução de novas estratégias terapêuticas, inclusive de prevenção do declínio cognitivo. O tratamento atual da demência associada à DP é apenas sintomático e geralmente traz resultados modestos.

\section{Materiais e métodos}

A redação desta revisão teve como ponto inicial a leitura de livros-texto, que permitiu a identificação dos domínios cognitivos mais comprometidos em pessoas com DP, com ou sem demência, sendo estes: funções executivas, habilidades visuoespaciais, memória, atenção e linguagem (Dekosky et al., 2004; Kavanagh e Marder, 2005). Os domínios cognitivos primeiramente identificados foram confirmados como os mais afetados na DP, pelos dados obtidos nos artigos revisados. Assim foram definidos os unitermos para a pesquisa, sendo os principais: demência, atenção, cognição, déficits cognitivos, funções executivas, fluência verbal, circuito fronto-estriatal, memória operacional, disfunções visuoespaciais, linguagem, inibidores da colinesterase, fisiopatologia, tratamento. Esses termos foram combinados com o unitermo doença de Parkinson. Assim, realizou-se revisão abrangente, embora de caráter não sistemático, que utilizou o Medline como base de busca de artigos.

Procuraram-se ensaios clínicos randomizados, estudos de coorte, caso-controle e revisões de literatura, com preferência por estudos datados a partir de 2000, porém estudos mais antigos foram incluídos conforme sua atual relevância.

\section{Fisiopatologia da demência na doença de Parkinson}

A modificação patológica mais marcante da DP encontra-se na substância negra pars reticulata. Esta se torna pálida a olho nu. Modificações microscópicas incluem perda de neurônios, gliose e os neurônios sobreviven- 
tes podem conter corpos de Lewy. Estes consistem em inclusões citoplasmáticas formadas principalmente por agregados protéicos. Mas a perda de neurônios, embora seletiva, não ocorre apenas na substância negra pars reticulata, abrangendo núcleos aminérgicos do tronco encefálico, núcleo basal de Meynert, núcleos hipotalâmicos e bulbo olfatório (Lang e Lozano, 1998). A causa da perda de neurônios não foi ainda definida, mas acredita-se que fatores genéticos, ambientais e acúmulo de proteínas estejam envolvidos.

O estudo de Braak et al. (2003) sugere uma ordem ascendente nas modificações patológicas da DP, que se desenvolve em células nervosas suscetíveis a essas alterações. Nesse estudo, os autores demonstraram que as lesões inicialmente ocorrem em certas áreas do tronco encefálico e no núcleo olfatório anterior. Na seqüência da evolução da moléstia, neurônios da substância negra são afetados e, posteriormente, áreas corticais passam a ser acometidas. Dessa forma, os autores admitem que a doença ascende, ao longo do tempo, do tronco encefálico às regiões corticais. $\mathrm{O}$ envolvimento cortical começa ântero-medialmente no mesocórtex temporal e, deste, para o neocórtex, atingindo os córtices associativos e áreas pré-frontais. Esse padrão de acometimento ascendente justificaria em parte a instalação mais tardia da demência na DP.

Pelo perfil dos déficits cognitivos provocados pela DP, comprometendo domínios de responsabilidade do lobo frontal, pode-se inferir que a disfunção desse lobo é causa de certas características das perdas cognitivas da DP, como déficit de memória operacional e queda de desempenho das funções executivas. Corroborando com essa teoria, estudos recentes de neuroimagem funcional demonstraram redução no metabolismo em áreas frontais durante recrutamento dos neurônios dessa região (Carbon e Marie, 2003). A disfunção do lobo frontal pode decorrer da perda de neurônios dopaminérgicos da substância negra, à medida que projeções da substância negra para o corpo estriado ficam comprometidas, reduzindo, assim, a atividade da alça fronto-estriatal, e também pela diminuição da atuação das projeções dopaminérgicas da área tegmental ventral para os lobos frontais e para o corpo estriado (Javoy-Agid e Agid, 1980; Pillon et al., 2003).

Em interessante estudo, Cools et al. (2002) demonstraram que a administração de levodopa aumenta o fluxo sanguiíneo no lobo pré-frontal dorsolateral direito e, assim, essa droga pode trazer melhora na cognição. Esses achados correlacionam-se com os resultados do estudo de Lange et al. (1992), que demonstraram piora no desempenho de funções cognitivas integradas no lobo frontal (memória operacional especial, planejamento no teste da Torre de Londres e paradigma de discriminação visual que inclui testes de mudança de atenção seletiva) após suspensão da terapia com levodopa.

A redução dopaminérgica também pode comprometer a atenção dos pacientes com DP. Corrêa (2006), ao verificar redução na latência no tempo de reação simples e redução na variação do tempo de reação com escolha em pacientes com DP, sem demência, após administração de levodopa, demonstrou que a dopamina proporciona melhora na identificação de estímulos e/ ou da resposta perante um estímulo. Assim sendo, a reposição de levodopa pode melhorar a atenção e/ou funções executivas, envolvidas nos ganhos cognitivos observados no estudo.

A associação de demência com gravidade do comprometimento motor sugere que mecanismos comuns são responsáveis pelas causas dessas manifestações da DP e que, portanto, contribui para a hipótese de que a disfunção dopaminérgica nigro-estriatal seja uma das causas do declínio cognitivo. Tal suposição é corroborada por um estudo de Holthoff-Detto et al. (1997), que utilizaram tomografia com emissão de pósitrons como forma de mensurar a relação da disfunção dopaminérgica com sintomas motores e declínio cognitivo em pacientes com DP. Entretanto, estudos de seguimento clínico de longo prazo mostram que o declínio cognitivo está apenas parcialmente relacionado ao comprometimento motor, indicando que outros mecanismos estão envolvidos, como veremos adiante (Marder et al., 1995; Portin e Rinne, 1986).

As alterações nos núcleos do prosencéfalo basal encontradas na DP (por exemplo, núcleo basal de Meynert) podem diminuir a atividade cortical em um padrão mais difuso, porém por redução da ação da acetilcolina (Carbon e Marie, 2003). Essas alterações das projeções colinérgicas para o córtex cerebral também contribuem para as disfunções executivas e, subseqüentemente, para o desenvolvimento da demência associada à DP (Perry et al., 1993). Degenerações, acometendo de modo marcante a região medial da substância negra, núcleos da base, amígdala, tálamo e locus ceruleus, também foram implicadas na gênese da demência da DP (Rinne et al., 1989; de la Monte et al., 1989; Perry et al., 1985; Zweig et al., 1993).

Apesar dos conhecimentos expostos anteriormente, os mecanismos neurobiológicos implicados na demência da DP ainda não estão por completo elucidados, tampouco são restritos à disfunção do lobo frontal, conforme referido. Dados anatomopatológicos sugerem que devem participar desses processos elementos neuropatológicos como os encontrados na doença de Alzheimer e a presença de corpos de Lewy no córtex cerebral (Becker et al., 1997; Emre, 2003a e b). Mattila et al. (1998) e Hurtig et al. (2000) demonstraram relação entre achados de corpos de Lewy em córtex cerebral e perda cognitiva na DP.

\section{Fatores de risco para o desenvolvimento de demência associada à doença de Parkinson}

Os resultados do estudo de Aarsland et al. (2001) indicam que pacientes com DP têm chance quase seis vezes 
maior de desenvolver demência do que a população de mesma idade. Em alguns estudos, avaliaram-se fatores de risco para o desenvolvimento de demência associada à DP e os resultados são por vezes conflitantes. Enumeramos a seguir fatores de risco encontrados em estudos mais recentes (Aarsland et al., 2001; Aarsland, 2003b; Hobson e Meara, 2004; de Lau et al., 2005; Huang et al., 2006; Woods e Troster, 2003; Janvin et al., 2006):

1. Pontuação na Escala de Hoehn e Yahr (empregada para estadiamento motor da DP) superior a 2 pontos.

2. Pontuação no Mini-Exame do Estado Mental (Folstein et al., 1975) inferior a 29 pontos.

3. Idade avançada.

4. Alucinações induzidas por levodopa.

5. Parkinsonismo com predomínio de bradicinesia ou padrão misto: tremor e bradicinesia.

6. Disfunção do lobo frontal.

7. Disfunção cognitiva em domínios que não a memória ou em múltiplos domínios cognitivos.

8. Portadores de ao menos um alelo E4 do gene apolipoproteína E (APOE E4) e, especialmente, do alelo e2 (APOE e2).

Burn et al. (2006) sugerem que a instabilidade postural precoce possa ser fator de risco para perda cognitiva em doentes com DP. Instabilidade postural é um sintoma da DP que responde pouco ao tratamento com levodopa, o que indica que deficiências de outros neurotransmissores diferentes da dopamina estão implicadas em sua gênese. Essas outras deficiências podem estar relacionadas a mecanismos patogênicos também implicados no aparecimento da demência na DP, postulam esses autores. Assim, a instabilidade postural precoce pode ser marcador para o desenvolvimento de demência associada à DP.

Estudos prospectivos sugerem que os pacientes que desenvolveram DP em idade mais avançada têm maiores riscos de desenvolver demência do que os indivíduos que manifestaram a moléstia mais precocemente (Mahieux et al., 1998; Caparros-Lefebvre et al., 1995; Foltynie et al., 2004). No entanto, essa relação não é confirmada por outros estudos (Hughes et al., 2000; Marder et al., 1995).

Alguns autores admitem que a depressão seja um fator de risco para o desenvolvimento de demência associada à DP (Mayeux et al., 1992). Aarsland et al. (2003a) observaram que depressão grave foi diagnosticada com maior frequiência nos pacientes que vieram a desenvolver demência. Porém, nesse mesmo estudo, a depressão não foi identificada como fator de risco independente para declínio cognitivo.

Outros possíveis fatores de risco encontrados em estudos da década de 1990 são: duração maior da DP (Biggins et al., 1992), deficiência em desempenho verbal na escala de inteligência de Wechsler (Biggins $e t$ al., 1992), doença cardiovascular (Salganik e Korczyn,
1990), história familiar de demência (Marder et al., 1990) e baixo nível educacional (Marder et al., 1994).

\section{Alterações cognitivas na doença de Parkinson em indivíduos sem demência}

Pode-se observar alterações em domínio cognitivo em pacientes com sintomas iniciais da DP, ou seja, em doentes sem demência e com manifestações motoras leves (Foltynie et al., 2004). O estudo de Muslimovic et al. (2005) confirma essa idéia ao demonstrar que déficits cognitivos proeminentes nos domínios da memória e das funções executivas são encontrados em pessoas com DP sem demência e ainda nas fases iniciais da enfermidade. Janvin et al. (2003) demonstraram que mais de 50\% dos pacientes com DP sem demencia têm alguma forma de alteração cognitiva e 20\% exibem predominantemente déficits de memória, 30\% sofrem de disfunções executivas e $50 \%$ têm desempenho cognitivo globalmente prejudicado.

Os pacientes com DP sem demência demonstram dificuldade em compreensão de sentenças gramaticalmente complexas (Grossman et al., 1991; Lieberman et al., 1990). Grossman et al. (2003), em estudo com ressonância magnética funcional, demonstraram que os pacientes com DP, sem demência, ao responderem a questões escritas, têm menor atividade neural nas regiões do estriado, da área pré-frontal ântero-medial e do lobo temporal direito. A dificuldade na compreensão de frases, portanto, pode surgir por interrupção de redes neurais importantes para processos cognitivos (como memória operacional e velocidade de processamento de informação) (Grossman et al., 2002; Lee et al., 2003) implicados no entendimento de frases, pois as regiões encefálicas que integram esses processos são menos ativadas durante a compreensão gramatical. Algumas dessas regiões fazem parte do circuito fronto-estriatotalâmico e a deficiência de neurotransmissores, como a dopamina, diminui a atividade desse circuito.

Outro transtorno envolvendo comunicação, a fala repetitiva, semelhante à disritmolalia ou à palilalia, pode ser encontrado em pacientes sem demência. Fatores lingüísticos com déficits no controle motor da fala podem produzir essa alteração na comunicação (Benke et al., 2000).

Outra alteração cognitiva que pode estar presente em pacientes com DP sem demência é a diminuição da capacidade de simulação e de abstração, atribuível à disfunção do lobo frontal (McNamara et al., 2003), possivelmente por redução do funcionamento do circuito fronto-estriato-talâmico.

Também se investigou a atenção em pacientes com DP, e estudos indicam que algumas áreas seletivas da atenção estão mais bem preservadas que outras. Como exemplo, a atenção sustentada e o desempenho no teste de extensão de dígitos (digit span) são normais. Por outro lado, o desempenho em testes de atenção, que depen- 
dem da velocidade de processamento cognitivo ou que requeiram que o paciente guie seus recursos de atenção, parece estar prejudicado (Zgaljardic et al., 2003).

Indivíduos com DP não medicados demonstram dificuldade em mudar o foco de atenção quando submetidos a diferentes estímulos visuais. Entretanto, drogas antiparkinsonianas reduzem esse déficit (Hayes et al., 1998). O tempo para iniciar movimentos simples em resposta aos estímulos visuais está aumentado em pacientes com DP, quando esses estímulos surgem entre outros que os distraem (Oliveira et al., 1998). Essas anormalidades sugerem que o controle voluntário da atenção nos parkinsonianos encontra-se comprometido em virtude da interrupção da ativação frontal exercida pelos núcleos da base, que é intermediada por projeções dopaminérgicas.

A visão também se encontra afetada em pacientes com DP. A deficiência dopaminérgica na retina provoca redução na percepção de contraste espacial. As alterações corticais também podem acarretar comprometimento visual e, em especial, déficits visuoespaciais podem ser diagnosticados em pacientes sem demência. Esses déficits podem ser atribuídos à disfunção executiva, motivados por interrupção dos sistemas frontoparietal e frontobasal, e não a uma genuína deficiência visual (Crucian et al., 2000). Memória visual e habilidades visuoconstrutivas já tiveram seu comprometimento demonstrado na DP e foram correlacionadas com instabilidade postural e dificuldade à marcha (Uc et al., 2005).

\section{Demência na doença de Parkinson}

O quadro demencial na DP instala-se em fases mais adiantadas da evolução da moléstia e tem como principais características a lentificação do processo cognitivo, a apatia, o comprometimento da memória e das funções executivas frontais (Bayles et al., 1996; Emre, 2003a e b).

A identificação de alterações cognitivas na DP oferece algumas dificuldades, visto que as funções executivas, que representam os domínios cognitivos geralmente afetados na DP, habitualmente não são avaliadas. São, portanto, necessários testes específicos para examinar essas funções que compreendem: formação de conceitos, solução de problemas, capacidade (aptidão) para mudança de padrões e elaboração de estratégias.

Os critérios do DSM-IV (Manual de Diagnóstico e Estatística de Doenças Mentais) normalmente empregados para caracterizar demências estão mais voltados para o diagnóstico de doença de Alzheimer e desconsideram que uma grave dificuldade motora compromete a autonomia do parkinsoniano. As dificuldades motoras nos parkinsonianos levam a superestimar o comprometimento cognitivo.

De acordo com revisão realizada por Miyasaki et al. (2006), poucos estudos foram formulados para avaliar a eficiência de escalas cognitivas para fazer o diagnóstico de demência em pacientes com DP. Segundo recomendações desses autores, o Mini-Exame do Estado Mental e o Exame Cognitivo de Cambridge (Cambridge Cognitive Examination [CAMCog]) são recursos úteis para rastreamento de demência em pacientes com DP. Nota-se que ambos têm sensibilidade equiparável, mas o CAMCog é mais específico por conter mais itens, embora requeira tempo maior para sua aplicação.

Algumas escalas foram desenvolvidas especialmente com o objetivo de avaliar demência associada à DP, levando-se em consideração as peculiaridades dessa complicação da doença. Entre essas, citamos a Escala para Resultados de Cognição na Doença de Parkinson (Scales for Outcomes of Parkinson's Disease-Cognition [SCOPA-COG]) (Marinus et al., 2003) e o Teste MiniMental Parkinson (Mahieux et al., 1995) que foram elaborados com a intenção de se verificar os domínios cognitivos mais comumente afetados pela DP.

A memória é secundariamente comprometida, e o armazenamento de novas informações está relativamente preservado, notando-se que os pacientes têm melhor desempenho em reconhecimento do que na lembrança sem pistas (Boller e Cappa, 2001). Esse é o protótipo da demência associada à $\mathrm{DP}$, mas alguns pacientes podem apresentar-se com perda da memória como a principal alteração cognitiva. Nesses casos, presume-se que se trata de DP em associação com doença de Alzheimer.

A diferenciação da demência da DP deve ser feita em relação às seguintes condições: depressão, confusão mental, demência dos corpos de Lewy, hidrocefalia de pressão normal e doença de Alzheimer (Klatka et al., 1996).

\section{Tratamento da demência associada à doença de Parkinson}

Uma consequiência importante da demência na DP é a restrição quanto ao uso de drogas antiparkinsonianas, pois nessas circunstâncias são muito mais propensas a provocar efeitos colaterais neuropsiquiátricos. A implicação clínica decorrente é o controle precário das dificuldades motoras e a redução da sobrevida. Dessa forma, o manejo do quadro demencial na DP envolve uma rigorosa seleção dos antiparkinsonianos a serem empregados, evitando-se o uso principalmente de fármacos com ação anticolinérgica.

Estudos recentes sobre o efeito de drogas de ação colinérgica empregadas no tratamento da doença de Alzheimer, tais como rivastigmina, donezepil e galantamina, têm mostrado resultados favoráveis sobre as alterações cognitivas da DP, sem piora do quadro motor. A droga memantina, antagonista do receptor de glutamato, também se mostra adequada para o tratamento da demência associada à DP (Bullock e Cameron, 2002; Aarsland et al., 2003; Kurita et al., 2003; Litvinenko et al., 2005; Aarsland et al., 2002; Ravina et al., 2005; Emre, 2004; Aarsland, 2004). 
A deficiência colinérgica na perda cognitiva da DP indica que os anticolinesterásicos podem ser usados e dados recentes sustentam essa hipótese. É importante mencionar que a rivastigmina foi aprovada pela FDA e pela Anvisa para o tratamento da demência associada à DP. Emre (2004), em artigo de revisão, afirma que as drogas anticolinesterásicas podem trazer melhora na fluência verbal, diminuição de alucinação, mais eficiência no desempenho de memória e também da atenção e da velocidade psicomotora.

A galantamina, em estudo não controlado com 16 pacientes, mostrou ser eficaz em proporcionar melhora cognitiva e em reduzir psicose, porém alguns pacientes apresentaram piora do tremor (Aarsland et al., 2003a).

Em dois ensaios clínicos randomizados e controlados com placebo, o donezepil mostrou ser bem tolerado e não piorou o parkinsonismo em doentes com demência associada à DP, além de proporcionar melhora cognitiva discreta (Aarsland et al., 2002; Ravina et al., 2005). No entanto, vale ressalvar que esses estudos incluíram número reduzido de pacientes (22 e 14 pacientes, respectivamente), o que limita suas conclusões.

Em ensaio clínico duplo-cego que randomizou 541 pacientes com demência associada à DP (dos quais 410 completaram estudo), Emre e Aarsland (2004) compararam os efeitos de rivastigmina com placebo por 24 semanas e observaram que a droga produziu melhora moderada, mas significante da cognição. Entretanto, o uso de rivastigmina associou-se à maior frequiência de vômitos, náusea e tremor. Uma extensão desse trabalho foi recentemente publicada (Poewe et al., 2006), demonstrando que os efeitos benéficos da terapia com rivastigmina prolongam-se por mais 24 semanas.

Wesnes et al. (2005) demonstram que a rivastigmina traz benefícios significantes e marcantes na atenção em pacientes com demência associada à DP. Essa conclusão sugere que, se analisarmos domínios específicos de cognição, poderemos encontrar melhoras cognitivas em maior escala.

Litvinenko et al. (2005) compararam os efeitos terapêuticos da memantina contra placebo em doentes com demência associada à DP por um período de 30 meses e concluíram que a droga traz benefícios cognitivos, diminui sintomas psicóticos e não afeta o desempenho motor dos doentes.

\section{Conclusão}

A DP não compromete apenas o sistema motor do paciente, mas, entre outras manifestações, provoca alterações cognitivas que podem estar presentes logo no início da doença. Essas alterações podem progredir com o avançar do tempo, configurando um quadro de demência. A demência associada à DP é altamente prevalente e causa significativa redução da qualidade de vida. O melhor conhecimento sobre fatores de risco e maior compreensão sobre os mecanismos biológicos envolvidos na demência associada à DP poderão implicar alguma forma de prevenção do desenvolvimento das alterações cognitivas.

A fisiopatologia da demência associada à DP não está completamente elucidada, mas são creditadas como responsáveis para sua instalação: a diminuição da atividade do lobo frontal decorrente da diminuição de aferências dopaminérgicas, a degeneração de núcleos colinérgicos subcorticais, a presença dos corpos de Lewy corticais e de elementos neuropatológicos da doença de Alzheimer.

As alterações cognitivas marcantes da demência associada à DP são déficits visuoespaciais, alterações de memória e disfunção cognitiva associadas ao mau funcionamento do lobo frontal, traduzido como comprometimento da memória operacional e das funções executivas. Esse mosaico de deficiências neuropsicológicas gera um peculiar perfil de comprometimento cognitivo, que deve ser avaliado com instrumentos adequados. $\mathrm{O}$ tratamento da demência mediante a utilização de drogas anticolinesterásicas ou da memantina demonstra ser seguro, mas proporciona resultados apenas modestos. Efeitos adversos que podem ser comumente encontrados são piora de tremor, náuseas e vômitos.

\section{Referências}

Aarsland, D.; Hutchinson, M.; Larsen, J.P. - Cognitive, psychiatric and motor response to galantamine in Parkinson's disease with dementia. Int $J$ Geriatr Psychiatry 18:937-941, 2003a.

Aarsland, D.; Laake, K.; Larsen, J.P.; Janvin, C. - Donepezil for cognitive impairment in Parkinson'sdisease: a randomised controlled study. J Neurol Neurosurg Psychiatry 72:708-712, 2002.

Aarsland, D. et al. - Mental symptoms in Parkinson's disease are important contributors to caregiver distress. Int J Geriatr Psychiatry 14:866-874, 1999.

Aarsland, D. et al. - Risk of dementia in Parkinson's disease: a communitybased, prospective study. Neurology 56:730-736, 2001.

Aarsland, D. et al. - Prevalence and characteristics of dementia in Parkinson's disease: an 8-year prospective study. Arch Neuro/60:387-392, 2003b.

Barbosa ER, Lefèvre BH, Comerlatti LR, Scaff M, Canelas HM. - Disfunções neuropsicológicas na doença de Parkinson. Estudo de 64 casos. Arq Neuro-Psiquiatr 45:109-118, 1987.

Barbosa, M.T. et al. - Parkinsonism and Parkinson's disease in the elderly: a community-based survey in Brazil (the Bambui study). Mov Disord 21:800-808, 2006.

Bayles, K.A. et al. - Change in cognitive function in idiopathic Parkinson's disease. Arch Neuro/ 53:1140-1146, 1996.

Becker, T. et al. - Parkinson's disease and depression: evidence for an alteration of the basal limbic system detected by transcranial sonography. J Neurol Neurosurg Psychiatry 63:590-596, 1997.

Benke, T.H; Hohenstein, C; Poewe, W; Butterworth, B. - Repetitive speech phenomena in Parkinson's disease. Neurol. Neurosurg. Psychiatry 69: 319-324, 2000.

Biggins, C.A. et al. - A controlled, longitudinal study of dementia in Parkinson's disease. J Neurol Neurosurg Psychiatry 55:566-571, 1992.

Boller, F.; Cappa, S. Cognitive deficits and dementia in Parkinson's disease. Handbook of neuropsychology. 2nd ed. Elsevier Sciences BV, Amsterdam, pp. 311-337, 2001.

Braak, H. et al. - Staging of brain pathology related to sporadic Parkinson's disease. Neurobiol Aging 24:197-211, 2003.

Bronnick, K. et al. - Neuropsychiatric disturbances in Parkinson's disease clusters in five groups with different prevalence of dementia. Acta Psychiatr Scand 112: 201-207, 2005. 
Bullock, R.; Cameron, A. - Rivastigmine for the treatment of dementia and visual hallucinations associated with Parkinson's disease. Curr Med Res Opin 18: 258-264, 2002.

Burn, D.J. et al. - Motor subtype and cognitive decline in Parkinson's disease, Parkinson's disease with dementia, and dementia with Lewy bodies. J Neurol Neurosurg Psychiatry 77:585-589, 2006.

Caparros-Lefebvre, D. et al. - Which factors predict cognitive decline in Parkinson's disease? J Neurol Neurosurg Psychiatry 58:51-55, 1995.

Carbon, M.; Marie, R.M. - Functional imaging of cognition in Parkinson's disease. Curr Opin Neurol 16:475-480, 2003.

Cools, R.; Stefanova, E.; Barker, R.A.; Robbins, T,W.; Owen, A.M. - Dopaminergic modulation of high-level cognition in Parkinson's disease: the role of the prefrontal cortex revealed by PET. Brain 125:584-594, 2002.

Corrêa, Y. Flutuação da atenção na doença de Parkinson. Tese de doutorado. Faculdade de Medicina da Universidade de São Paulo, 2006.

Cummings, J.L. - Intellectual impairment in Parkinson's disease: clinical, pathologic, and biochemical correlates. J Geriatr PsychiatryNeurol 1:24-36, 1988.

Crucian, G.P. et al. - Cognitive and vestibulo-proprioceptive components of spatial ability in Parkinson's disease. Neuropsychologia 2000; 38:757-767.

De La Monte, S.M.; Wells, S.E.; Hedley-Whyte, E.T.; Growdon, J.H. - Neuropathological distinction between Parkinson's dementia and Parkinson's plus Alzheimer's disease. Ann Neurol 26:309-320, 1989.

De Lau, L.M.; Schipper, C.M.; Hofman, A.; Koudstaal, P.J.; Breteler, M.M. - Prognosis of Parkinson disease: risk of dementia and mortality: the Rotterdam Study. Arch Neurol 62:1265-1269, 2005.

Dekosky, S.T; Kaufer, D.I; Loez, O.L. - The dementias. In: Bradley GW, Daroff RB, Fenichel GM, Jankovic, J (eds.) Neurology in Clinical Practice. Vol. II. Elsevier Inc, pp. 1924-1926, 2004.

Emre, M. - Dementia associated with Parkinson's disease. Lancet Neurol 2: 229-237, 2003a.

Emre, M. - What cause mental dysfunction in Parkinson's disease? Mov Disord 18 (suppl 6): S63-S71, 2003b.

Emre, M. - Dementia in Parkinson's disease: cause and treatment. Curr Opin Neurol 17: 399-404, 2004.

Emre, M.; Aarsland, A. - Rivastigmine for dementia associated with Parkinson's disease. N Engl J Med 351: 2509-2518, 2004.

Folstein, M.F.; Folstein, S.E.; Mchugh, P.R. - Mini-mental state. A practical method for grading the cognitive state of patients for the clinician. J Psychiatr Res 12:189-198, 1975.

Foltynie, T.; Brayne, C.E.; Robbins, T.W.; Barker, R.A. - The cognitive ability of an incident cohort of Parkinson's patients in the UK: the CamPaIGN study. Brain 127:550-560, 2004.

Grossman, M.; Carvell, S.; Gollomp, S.; Stern, M.B.; Vernon, G.; Hurtig, H.I. - Sentence comprehension and praxis deficits in Parkinson's disease. Neurology 41:1620-1626, 1991.

Grossman, M. et al. - Information processing speed and sentence comprehension in Parkinson's disease. Neuropsychology 16: 174-181, 2002.

Grossman, M. etal. - Grammatical and resource components of sentence processing in Parkinson's disease - An fMRI study. Neurology60:775-781, 2003.

Hayes, A.E.; Davidson, M.C.; Keele, S.W.; Rafal, R.D. Toward a functional analysis of the basal ganglia. J Cogn Neurosci 10:178-198, 1998.

Hobson, P.; Meara, J. - Risk and incidence of dementia in a cohort of older subjects with Parkinson's disease in the United Kingdom. Mov Disord 19:1043-1049, 2004.

Holthoff-Detto, V.A. et al. - Functional effects of striatal dysfunction in Parkinson disease. Arch Neurol 54:145-150, 1997.

Huang, X.; Chen, P.; Kaufer, D.I.; Troster, A.I.; Poole, C. - Apolipoprotein $\mathrm{E}$ and dementia in Parkinson disease: a meta-analysis. Arch Neurol 63(2):189-193. 2006

Hughes, T.A. et al. - A 10-year study of the incidence of and factors predicting dementia in Parkinson's disease. Neurology 54:1596-1602, 2000.

Hurtig, H.I. et al. - Alpha-synuclein cortical Lewy bodies correlate with dementia in Parkinson's disease. Neurology 54:1916-1921, 2000.

Jacobs, D. M. et al. - Neuropsychological characteristics of preclinical dementia in Parkinson's disease. Neurology 45:1691-1696, 1995.

Janvin, C.; Aarsland, D.; Larsen, J.P.; Hugdahl, K. - Neuropsychological profile of patients with Parkinson's disease without dementia. Dement Geriatr Cogn Disord 15: 126-131, 2003.
Janvin, C.C.; Larsen, J.P.; Aarsland, D.; Hugdahl, K. - Subtypes of mild cognitive impairment in Parkinson's disease: progression to dementia. Mov Disord 23: 2006.

Javoy-Agid, F.; Agid, Y. - Is the mesocortical dopaminergic system involved in Parkinson's disease? Neurology 30:1326-1330, 1980.

Kavanagh, P.; Marder, K. - Dementia. In: Pfeiffer, R.F; Bodis-Wollner, I. (eds.) Parkinson's disease and non motor dysfunction. Humana Press, New Jersey, pp. 35-45, 2005.

Klatka, L.A.; Louis, E.D.; Schiffer, R.B. - Psychiatric features in diffuse Lewy body disease: a clinicopathologic study using Alzheimer's disease and Parkinson's disease comparison groups. Neurology 47:1148-1152, 1996.

Kurita, A.; Ochiai, Y.; Kono, Y.; Susuk, M.; Inoue, K. - The beneficial effect of donepezil in visual hallucinations in three patients with Parkinson's disease. J Geriatr Psychiatry Neurol 16:184-188, 2003.

Lang, A.E.A.M.; Lozano, A.M. - Parkinson's disease - First of two parts. N Engl J Med 339: 1044-1053, 1998.

Lange, K.W.; Robbins, T.W.; Marsden, C.D.; James, M.; Owen, A.M.; Paul, G.M. - L-dopa withdrawal in Parkinson's disease selectively impairs cognitive performance in tests sensitive to frontal lobe dysfunction Psychopharmacology (Berl)107:394-404, 1992.

Lee, C.; Grossman, M.; Morris, J.; Stern, M.B.; Hurtig, H.I. - Attentional resource and processing speed limitations during sentence processing in Parkinson's disease. Brain Lang Brain Lang 85:347-356, 2003.

Lieberman, P.; Friedman, J.; Feldman, L.S. - Syntax comprehension in Parkinson's disease. J Nerv Ment Dis 178:360-366, 1990.

Litvinenko, I.V; Odinak, M.; Mogilnaya, V.I.; Shatova, A.V. - Therapy by memantine in demented patients with Parkinson's disease improves cognitive functions and preserves of motors functional abilities for 30 months period. Europ J Neurology Suppl.122:111, 2005.

Mahieux, F.; Michelet, D.; Manifacier, M.; Boller, F.; Fermanian, J.; Guillard A. - Mini-mental Parkinson: first validation study of a bedside test constructed for Parkinson's disease. Behav Neurol 8: 15-22, 1995.

Mahieux, F. et al. - Neuropsychological prediction of dementia in Parkinson's disease. J Neurol Neurosurg Psychiatry 64:178-183, 1998.

Marder, K.; Cote, L.; Tang, M. - The risk and predictive factors associated with dementia in Parkinson's disease. In: Korczyn, A. Dementia in Parkinson's disease. Monduzzi, Bologna, pp. 51-54, 1994.

Marder, K.; Flood, P.; Cote, L.J.; Mayeux, R. - A pilot study of risk factors for dementia in Parkinson's disease. Mov Disord 5:156-161, 1990.

Marder, K. et al. - The frequency and associated risk factors for dementia in patients with Parkinson's disease. Arch Neurol 52:695-701, 1995.

Marinus, J.; Visser, M.; Verwey, N.A.; Verhey, F.R.; Middelkoop, H.A.; Stiggelbout, A.M.; van Hilten, J.J. -Assessment of cognition in Parkinson's disease. Neurology 61:1222-1228, 2003.

Mattila, P.M. et al. - Cortical Lewy bodies and Alzheimer-type changes in patients with Parkinson's disease. Acta Neuropatho/ 95:576-582, 1998.

Mayeux, R.; Denaro, J.; Hemenegildo, N.; Tang, M.X.; Stern, Y. - A populationbased investigation of Parkinson's disease with and without dementia. Arch Neurol 49:492-497, 1992.

McKeith, I.G. et al. - Diagnosis and management of dementia with Lewy bodies: third report of the DLB Consortium. Neurology65:1863-1872, 2005.

McNamara, P.; Durso, R.; Brown, A.; Lynch, A. - Counterfactual cognitive deficit in persons with Parkinson's disease. J Neurol Neurosurg Psychiatry 74:1065-1070, 2003.

Miyasak, I.J.M. et al. - Practice parameter: evaluation and treatment of depression, psychosis, and dementia in Parkinson disease (an evidence-based review): report of the Quality Standards Subcommittee of the American Academy of Neurology. Neurology 66:996-1002, 2006.

Muslimovic, D. et al. - Cognitive profile of patients with newly diagnosed Parkinson disease. Neurology 65:1239-1245, 2005.

Oliveira, R.M.; Gurd, J.M.; Nixon, P.; Marshall, J.C.; Passingham, R.E. - Hypometria in Parkinson's disease. Automatic versus controlled processing. Move Disord 13:422-427, 1998.

Parkinson, J. An essay on the shaking palsy. Whittingham and Rowland for Sherwood, Neely and Jones, London, 1817.

Perry, E.K. et al. - Cholinergic correlates of cognitive impairment in Parkinson's disease: comparisons with Alzheimer's disease. J Neurol Neurosurg Psychiatry 48:413-421, 1985.

Perry, E.K. et al. - Cholinergic transmitter and neurotrophic activities in Lewy body dementia: similarity to Parkinson's and distinction from Alzheimer disease. Alzheimer Dis Assoc Disord 7:69-79, 1993. 
Pillon, B.; Czernecki, V.; Dubois, B. - Dopamine and cognitive function. Curr Opin Neurol 16 (Suppl 2):S17-22, 2003.

Poewe, W.; Wolters, E.; Emre, M.; Onofrj, M.; Hsu, C.; Tekin, S.; Lane, R. - Long-term benefits of rivastigmine in dementia associated with Parkinson's disease: an active treatment extension study. Mov Disord 2006; 21:456-461, 2006.

Portin, R.; Rinne, U.K. - Predictive factors for cognitive deterioration and dementia in Parkinson's disease. Adv Neurol 45:413-416, 1986.

Ravina, B.; Putt, M.; Siderowf, A.; Farrar, J.T.; Gillespie, M.; Crawley, A.; Fernandez, H.H.; Trieschmann, M.M.; Reichwein, S.; Simuni, T. - Donepezil for dementia in Parkinson's disease: a randomised, double blind, placebo controlled, crossover study. J Neurol Neurosurg Psychiatry 76:934-939, 2005.

Rinne, J.0.; Rummukainen, J.; Paljarvi, L.; Rinne, U.K. - Dementia in Parkinson's disease is related to neuronal loss in the medial substantia nigra. Ann Neurol 26:47-50, 1989.

Salganik, L.; Korczyn, A. - Risk factors for dementia in Parkinson's disease. Adv Neurol 53:343-347, 1990.

Schrag, A.; Jahanshahi, M.; Quinn, N. - What contributes to quality of life in patients with Parkinson's disease? J Neurol Neurosurg Psychiatry 69:308-312, 2000.
Thanvi, B.R.; Munshi, S.K.; Vijaykumar, N.L.O.T.C . - Neuropsychiatric non-motor aspects of Parkinson's disease. Postgrad Med J79:561-565, 2003.

Uc, E.Y. et al. - Visual dysfunction in Parkinson disease without dementia. Neurology 65:1907-1913, 2005.

Weintraub, D.; Moberg, P.J.; Duda, J.E.; Katz, I.R.; Stern, M.B. - Effect of psychiatric and other nonmotor symptoms on disability in Parkinson's disease. J Am Geriatr Soc 52:784-788, 2004.

Wesnes, K.A.; McKeith, I.; Edgar, C.; Emre, M.; Lane, R. - Benefits of rivastigmine on attention in dementia associated with Parkinson disease. Neurology 65:1654-1656, 2005.

Woods, S.P.; Troster, A.I. - Prodromal frontal/executive dysfunction predicts incident dementia in Parkinson's disease. $J$ Int Neuropsychol Soc 9:17-24, 2003.

Zgaljardic, D.J.; Borod, J; Foldi, N.S.; Mattis, P. - A review of the cognitive and behavioral sequelae of Parkinson's disease: relationship to frontostriatal circuitry. Cog Behav Neurol 16:193-210, 2003.

Zweig, R.M.; Cardillo, J.E.; Cohen, M.; Giere, S.; Hedreen, J.C. - The locus ceruleus and dementia in Parkinson's disease. Neurology 43: 986-991, 1993. 